\title{
Image Edge Detection and Segmentation by using Histogram Thresholding method
}

\author{
*Dr. V.S. Manjula, \\ Associate Professor Computer Science \& Engineering Department St. Joseph University College of Engineering \\ Technology Dar-Es-Salaam, Tanzania, East Africa \\ Corresponding Author: Dr. V.S. Manjula,
}

\begin{abstract}
A new approach used for image edge deduction, segmentation and normalization illumination under varying lighting conditions are presented. Edge detection refers to the process of identifying and locating sharp by applying smooth and noisy clinical technic in an image. It has favorable applications in the fields such as machine vision, pattern recognition, object recognition, motion analysis, pattern recognition, medical image processing \& biomedical imaging. Segmentation refers to the process of partitioning a digital image into the multiple segments using set of pixels as to simplify and change the representation of an image and easier to analyze. Edge detection highlights high frequency components in the image. Edge detection is becomes more arduous when it comes to noisy images. The study focuses on fuzzy concepts based edge detection in smooth and noisy clinical images. Traditional method of edge detection involves convolving the image with an operator (2-D filter) changes in pixel intensity scene which is constructed to be sensitive to large gradients. Edge detectors form a collection of different images and applying local image processing method to locate sharp changes in the intensity function. In this paper, histogram thresholding is proposed in order to help the edge detection and segmentation of image to found robust way regardless of the segmentation approach applying for histogram thresholding algorithm. This paper shows the comparison of edge detection \& segmentation techniques under different conditions \& variation of intensity pixel value of the selected algorithms.

Index terms: Edge deduction, Image Segmentation, Noise Histogram Equalization, Illumination Normalization, Fuzzy algorithm \& Histogram Thresholding.
\end{abstract}

\section{INTRODUCTION}

Edge detection is one of the most frequently used techniques in digital image processing. Edge detection method is a process of identifying and locating sharp discontinuities in an image. The boundaries of object surfaces discontinuities are rapid changes in pixel intensity which characterizes boundaries of objects in a scene. Edges in an image are contours generated as a result of sudden change of multiple characteristics at pixel level. These changes could be observed due to alteration in color, texture, shade or light absorption. These characteristics could further lead in estimating the size, orientation, depth and surface features in an image. In medical images the role of edge detection is significant and has extensively been employed for the detection of structures and anomalies in Computerized Tomography (CT) scans, Position Emission Tomography (PET) scans and Magnetic Resonance Images (MRI). The detected images embody high frequency noise or irrelevant data which inhibits the detection of continuous edge points, since edge itself is a composition of high frequency data. The noise generates false flags as they often mislead the algorithms for an edge.

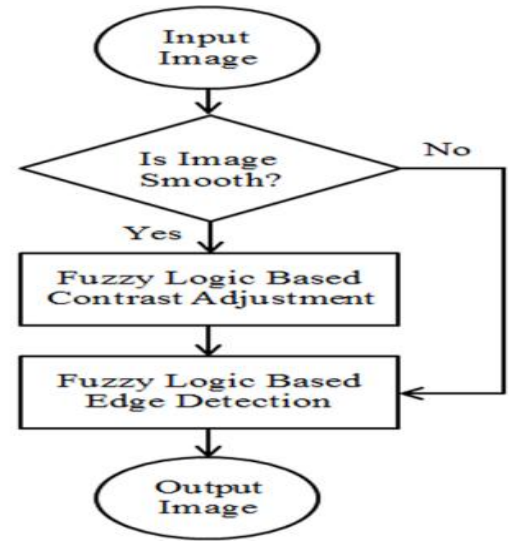

Fig1 Classification of edge detection

To perform image segmentation and edge detection tasks, there are many methods that incorporate region growing and edge detection techniques. For example, it is applying edge detection techniques to obtain region growing techniques to work and combining both special and intensity 
information in image segmentation approach based on multi-resolution edge detection, region selection and intensity threshold methods to detect gray matter structure. Algorithm are generalizing to include spatial constraints and to account for local intensity variations in the image. It designed and implemented an image based on region-growing techniques by applying segmentation method.

\section{EDGE DETECTION TECHNIQUES:}

Edge detection is an important technique of detecting and identifying by locating sharp discontinuities in an image. So in this method is a vital step in image analysis and solving many complex problems. The main aim of study edge detection for image segmentation using various computing approaches based on different techniques.

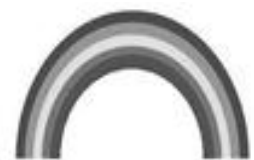

(a)

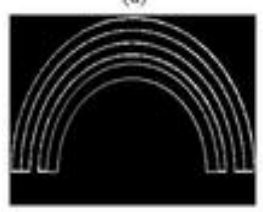

(d)

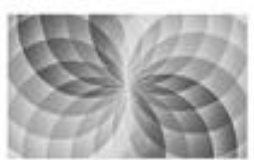

(b)

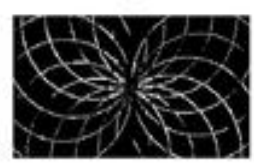

(c)

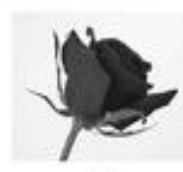

(c)

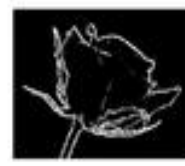

(f)
Fig2 Original image with edge detection

The boundaries of object surfaces in a scene often lead to oriented localized changes in intensity of an image called edges. Classical methods of edge detection involves convolving the image with an operator which is constructed to be sensitive to large gradients in the image while returning values of zero in uniform regions. Edge detection technique also transforms images. In image processing field are used in real medical images for testing and segmentation is a subset of an expansive field of Computer Vision which deals with the analysis of the spatial content of an image.

Laplacian: : The Laplacian method searches for zero crossing in the second derivative of the image to find edges and which combines median filter and simple standard deviation to accomplish an excellent edge detector for image processing Edge detection refers to the process of identifying and locating sharp discontinuities in an image. There are certain types of edges with an operator 2-D filter, which is constructed to be sensitive to large gradients in the image while returning values of zero in uniform regions. Variables involved in the selection of an edge detection operator include Edge orientation, Noise environment and Edge structure. The geometry of the operator determines a characteristic direction in which it is most sensitive to edges. The operator needs to be optimized to look for horizontal, vertical, or diagonal edges. Edge detection is difficult to implement in noisy images, since both the noise and the edges contain high frequency content. In attempts to reduce the noise result in blurred and distorted edges. Operators used on noisy images are typically larger in scope, so they can average enough data to discount localized noisy pixels. Hence the comparison of various edge detection techniques and analysis of image edges, edge localization and high computation time.

\section{IMAGE SEGMENTATION TECHNIQUES:}

Image segmentation methods are categorized on the basis of two properties discontinuity and similarity. Methods based on discontinuities are called as boundary based methods and similarity are called Region based methods. Segmentation is a process that divides an image into its regions or objects that have similar features or characteristics. Mathematically complete segmentation of an image $\mathrm{R}$ is a finite set of regions R1...Rn. Image segmentation methods can be categorized as Region Based methods \& Clustering methods.

\section{A. Region Based Techniques:}

Region based methods are based on continuity. These techniques divide the entire image into sub regions depending on some rules like all the pixels in one region must have the same gray level. Region-based techniques rely on common patterns in intensity values within a cluster of neighboring pixels. The cluster is referred to as the region, and the goal of the segmentation algorithm is to group the regions according to their anatomical or functional roles.

\section{B. Clustering Technique:}

In this methods is taken and then each point is added to the cluster where the difference between the point and the mean is smallest. Since clustering works on hue estimates it is usually used in dividing a scene into different objects. The performance of clustering algorithm for image segmentation is highly sensitive to features used and types of objects in the image and hence generalization of this technique is difficult. A new shape-based image segmentation algorithm called fuzzy clustering for image segmentation using generic shape information which integrates clustering framework. Hence using the algorithm presented and can be used for many different object shapes and hence one framework can be used for different applications like medical imaging, security systems and any image processing application where arbitrary shaped object segmentation is required. 


\section{Split and Merge Technique:}

There are two parts to this technique first the image is split depending on some criterion and then it is merged. The whole image is initially taken as a single region then some measure of internal similarity is computed using standard deviation. If too much variety occurs then the image is split into regions using thresholding. This is repeated until no more splits are further possible. Quad tree is a common data structure used for splitting comes the merging phase, where two regions are merged if they are adjacent and similar. Similarity can be measured by comparing the mean gray level or using statistical tests. Two regions $\mathrm{R} 1$ and $\mathrm{R} 2$ are merged into R3.

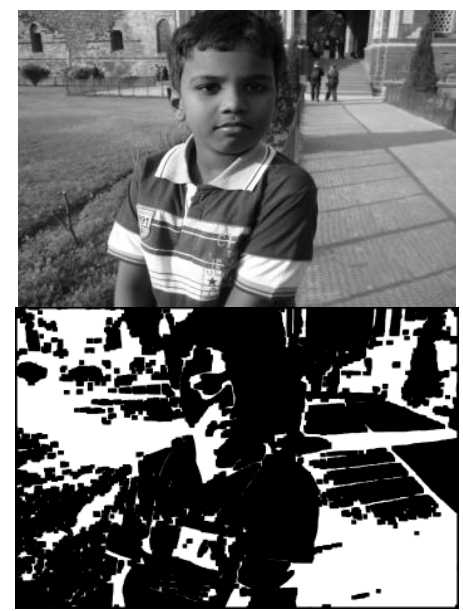

Fig3 Images of the same person region using thresholding

Where, $\mathrm{m} 1$ and $\mathrm{m} 2$ are the mean gray-level values in the regions $\mathrm{R} 1$ and $\mathrm{R} 2$, and $\mathrm{T}$ is threshold merging is repeated until no more further merging is possible. The major advantage of this technique is guaranteed connected regions.

\section{Thresholding:}

This is the simplest way of segmentation. Using thresholding technique regions can be classified on the basis range values, which is applied to the intensity values of the image pixels. Thresholding is the transformation of an input image (a) to an output (segmented) binary image (d) as follows.

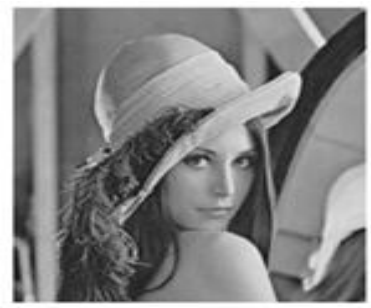

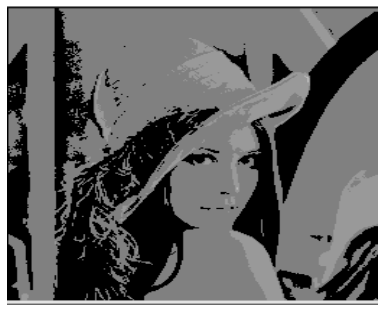

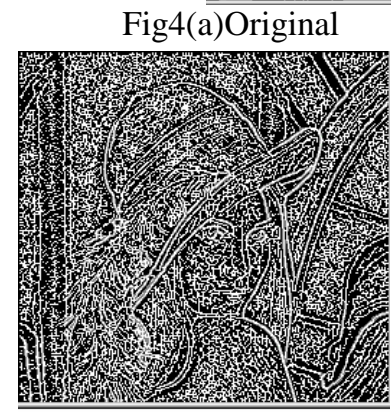

Fig4(c) with Threshold
Fig4 (b) edge detection

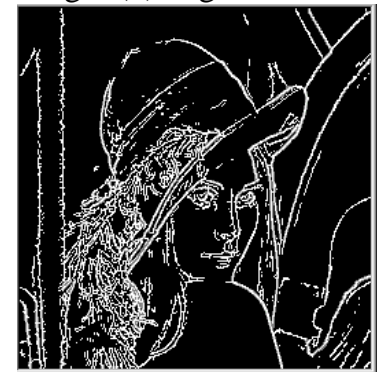

Fig4 (d) without Threshold
Where $\mathrm{T}$ is the threshold, $\mathrm{g}(\mathrm{i}, \mathrm{j})=1$ for image elements of objects and $g(i, j)=0$ for image elements of the background. Thresholding is computationally inexpensive and fast, it is the oldest segmentation method and is still widely used in simple applications. Using range values or threshold values, pixels are classified using either of the thresholding techniques like global and local thresholding. Global thresholding method selects only one threshold value for the entire image. Local thresholding selects different threshold values for different regions. To segment complex images multilevel thresholding is required.

\section{DISCUSSION \& COMPARISON OF EXPERIMENTAL RESULTS:}

Many techniques have been employed for the development of an optimum edge detection algorithm. Each effort is guided by the motivation to overcome the limitations in previous methodologies. The conventional techniques incorporate the use of linear time invariant filters. These filters recognize an edge as an abrupt change of grey scale pixel intensities. The techniques are well established and computationally efficient. Canny, Sobel \& Robert are based on the concept of spatial differential filters utilizing local gradient. These filters process the data in a relatively short time and are computationally optimized, however, they are susceptible to noise. The developed edge detection technique for noisy images is based on fuzzy logic. The image was designed to take the greyscale values of neighborhood pixels from the input image. The greyscale values of the neighborhood pixels obtained from the mask were pre-processed prior to the fuzzy inference system. The values were subsequently converted into the fuzzy plane. A fuzzy rule base was 
defined to determine and show the edge pixels' in the output image. The block diagram of the proposed fuzzy edge detection is shown in other segmentation techniques. However, their limitation was their inadaptability to regions with varying greyscale due to a fixed threshold point.

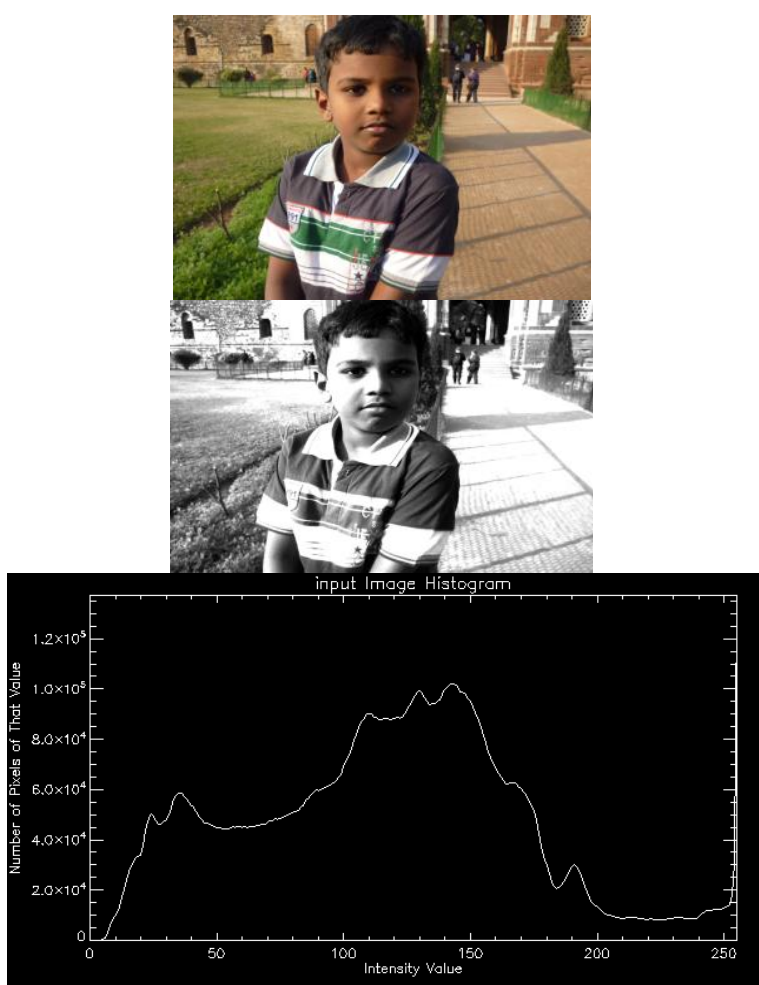

Fig5 Gray conversion with histogram

The developed methodology for edge detection followed by simulation of results. The proposed edge detection algorithm for noisy and clinical images is based on a fuzzy inference system. A two mask technique was used to detect edges in greyscale images. For detection of edges in noisy images only one mask (edge detection mask) was used. However, for smooth clinical images an extra mask of contrast adjustment was integrated with the edge detection mask to intensify the image based on fuzzy logic. The workflow of the proposed methodology is shown

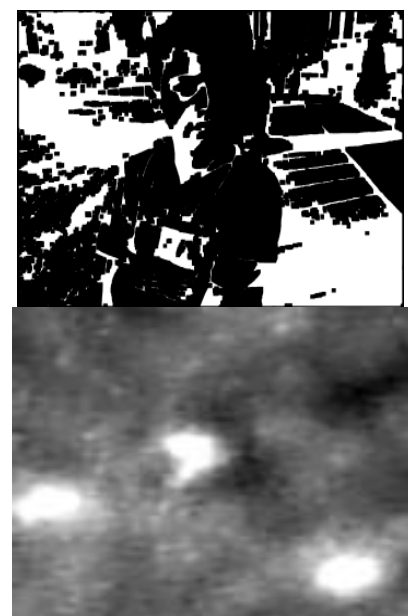

Fig6 edge detection of noisy imaes of the same person under different angle variation.

Illumination variation is one of intractable crucial problems in face recognition and many lighting normalization approaches have been proposed in the past decades. Most of the illumination corresponds to low frequency region. A scaling of low frequency DCT coefficients is utilized for normalization method, which performs adaptive preprocessing for each testing image according to its lighting attribute. The representative methods of this category are histogram equalization, gamma intensity correction \& logarithm transform. But these global processing techniques of image processing are found to be insufficient to overcome variations due to illumination changes.

A. Fuzzy Set: Fuzzy Set theory is another technique that has been employed for edge detection. The method performs mathematical and logical reasoning based on approximations rather than crisp values. Therefore the technique significantly reduces the complexity of problems where fixed values cannot be attained or predicted. The results for edge detection were appreciable in images without noise but performed poorly when noise was introduced.

\section{B. Simulation Results and Discussion:}

Recently many researchers have been published on illumination processing in face recognition. These approaches can be divided into two categories; one is active approach and another is passive approach. In active approach focuses the problem by employing active imaging techniques to obtain face images captured in consistent of illumination invariant modalities.

The proposed method (in noisy images) employs a $3 \times 3$ mask guided by fuzzy rule set. Moreover, in case of smooth clinical images, an extra mask of contrast adjustment is integrated with edge detection mask to intensify the smooth images. The developed method was tested on noise-free, smooth and noisy images. The results were compared with 
other established edge detection techniques like Sobel, Prewitt, Laplacian of Gaussian (LOG), Roberts and Canny. When the developed edge detection technique was applied to a smooth clinical image of size $270 \times 290$ pixels having $24 \mathrm{~dB}$ 'salt and pepper' noise, it detected very few false edge pixels, compared to Sobel, and Canny false edge pixels. Therefore it is evident that the developed method offers improved solution to the edge detection problem in smooth and noisy clinical images. The developed edge detection technique was tested on a number of greyscale images including noise free, noisy and smooth images. For noise free and noisy images, only one mask (Edge detection) was employed. However, for smooth clinical images contrast adjustment mask was collectively used, with edge detection mask.

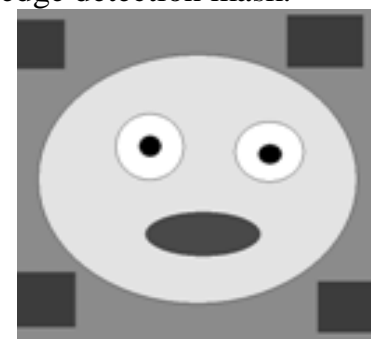

Fig 7(a)Original image

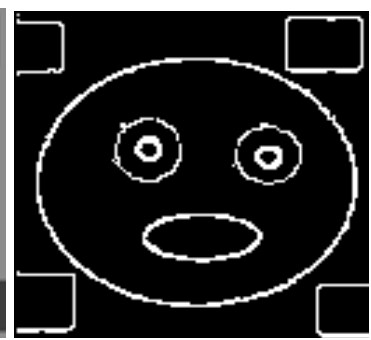

(b) median filter

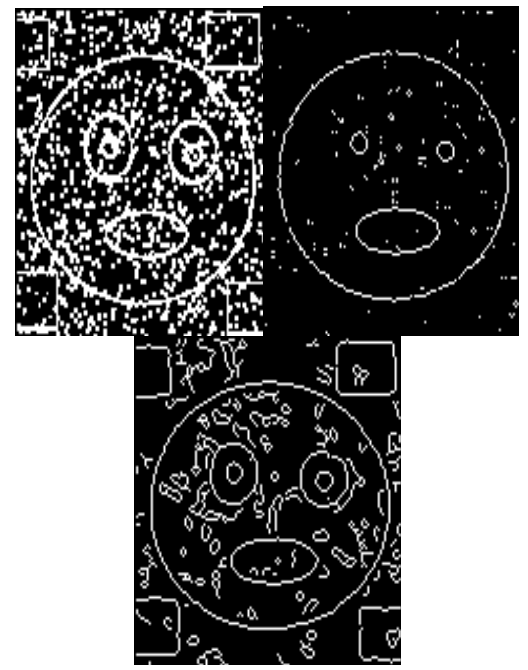

c) without median filterd)Sobel filter (e) Canny filter

Finally the developed edge detection technique was applied to the smooth clinical image of It is clear from results that the developed technique shows excellent results compared to the established edge detection techniques. The number of false edge pixels detected by various edge detection techniques, when subject to images having different intensity pixel values. It obvious that the number of false edge pixels detected by various techniques increased the noise level in the images. Statistical analysis such as sensitivity and specificity of the proposed technique with Sobel, Canny and scan line approximation. The proposed technique has potential applications in many disciplines ranging from medical MRI images like bones defects/cracks, industrial surface inspection, crack detection \& rust detection and in agriculture such as identification of deforestation, crop yield production, identification of nutritional deficiencies.

\section{Histogram Equalization:}

Histogram equalization maps the input image's intensity values so that the histogram of the resulting image will have an approximately uniform distribution.

The histogram of a digital image with gray levels in the range $[0, L-1]$ is a discrete function $\mathrm{p}(r k)$ $=n k / n$, where $r k$ is the $\mathrm{k}^{\text {th }}$ gray level, $n k$ is the number of pixels in the image with that gray level, $n$ is the total number of pixels in the image, and $k=0,1$, 2 , ..., $L-1$. Basically $\mathrm{p}(r k)$ gives an estimate of the probability of occurrence of gray level $r k$.

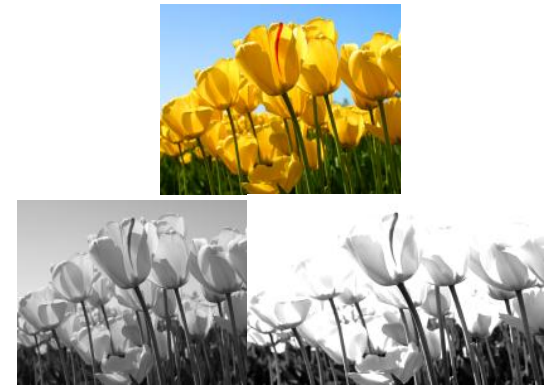

Fig 8. Equalized input image

In noise free greyscale images, the developed technique has successfully detected all type of edges as shown in figure 8. The proposed technique for edge detection have detected boundary lines (edges) successfully detected edges in the greyscale (flower) images as shown.

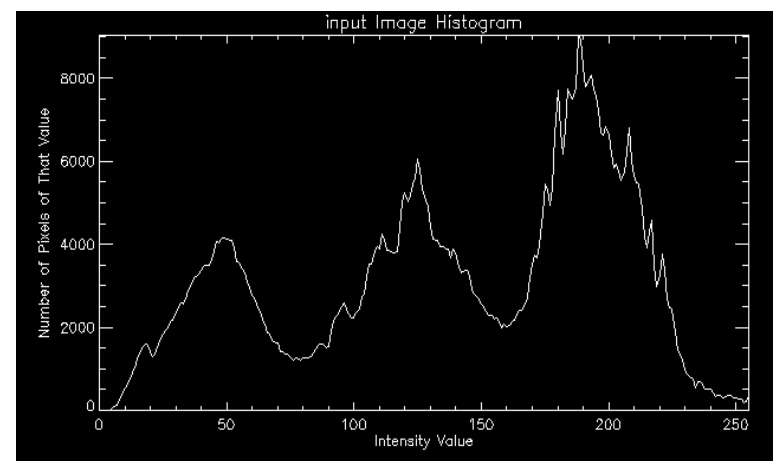

Fig 9 Equalized input image Histogram

\section{Gray image Thresholding Histogram:}

Histogram equalization, the local contrast of the object in the image is increased, especially when the applied data of the image is represented by close contrast values. The intensity can be better 
distributed on the histogram, through this adjustment. This allows for areas of lower local contrast to gain a higher contrast without affecting the global contrast. In histogram equalization, the goal is to obtain a uniform histogram for the output image, is an "optimal" overall contrast is perceived.

However, the feature of interest in an image might need enhancement locally. Adaptive Histogram Equalization (AHE) computes the histogram of a local window centered at a given pixel to determine the mapping for that pixel, which provides a local contrast enhancement.

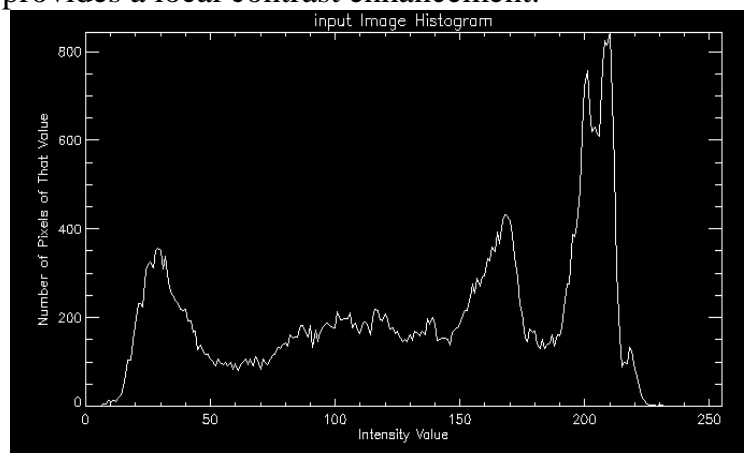

Fig10 Image Histogram equalization of original image

However, the enhancement is so strong that two major problems can arise: noise amplification in "flat" regions of the image and "ring" artifacts at strong edges. CLAHE operates on small regions in the image, (title) rather than entire image. Each tile's contrast is enhanced, so that the histogram of the output region approximately matches the histogram specified by a distribution parameter, which may be a uniform or a different shape histogram.

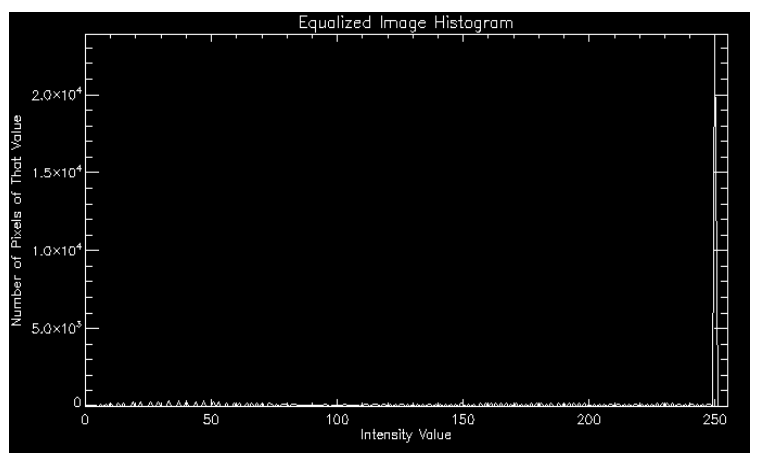

Fig11 Equalized input Images Histogram.

Coefficients and their selection in zigzag pattern. After applying adaptive histogram equalization, we employed logarithm transform] for further enhancement of the image.

\section{CONCLUSION}

We propose a new methodology and a framework for evaluating edge detection by simple statistical standard deviation had been presented. The proposed method was tested on binary level test images and compared for illumination normalization with two classical edge detectors which are Sobel and Canny. In this work histogram thresholding was proposed as a new way of improve image segmentation thresholding of the final histogram is done relatively easy and all it takes is definition of a low pass filters. Experiment results have demonstrated that the proposed algorithm for edge detection produces satisfactory results for different gray level digital images. The performance of the proposed approach is quite better for different illumination variation normalization is achieved under all illumination variations. Thus better illumination therefore, a good recommendation is that the proposed edge detector is suitable for Medical, Agriculture \& Biometric identification that is needed to obtain considerable better results during the segmentation process. The method has been successfully tested with segmentation methods for the proposed edge detector of image is easy to implementation.

\section{REFERENCES}

[1] Bovik A. C., Huang T. S., and Munson D. C. 1985. Edge-sensitive image restoration using order constrained least squares methods. IEEE Trans. Acoust., Speech, Signal Processing, vol. 33, pp. 1253-1263.

[2] Canny J 1986. A computational approach to edge detection. IEEE Transactions on Pattern Analysis and Machine Intelligence, 8(6):679-698.

[3] Desolneux A., Moisan L. And Morel J.-M. 2001. Edge Detection by Helmholtz Principle, Journal of Mathematical Imaging and Vision 14: 271-284.

[4] Frei W. and Chen C. 1977. Fast Boundary Detection: A Generalization and New Algorithm. IEEE Trans. Computers, vol. C26, no. 10, 988-998.

[5] Gao W., Yang L., Zhang X. and Liu H. 2010. An Improved Sobel Edge Detection. IEEE international conference on computer science and information technology (ICCSIT), vol. 5, 67-71.

[6] Giannarou S. and Stathaki T. 2011. Optimal edge detection using multiple operators for image understanding. EURASIP Journal on Advances in Signal Processing 2011, 28.

[7] Becerikli Y. and Karan T. M. 2005. A New Fuzzy Approach for Edge Detection. IWANN 2005, LNCS 3512, 943-951

[8] Tzu-Heng Henry Lee. Edge Detection Analysis. Graduate Institute of Communication Engineering, National Taiwan University, Taipei, Taiwan, ROC. 
[9] Yi S., Labate D., Easley G. R. and Krim H. 2009. A Shearlet Approach to Edge Analysis and Detection. IEEE Trans. Image Proc. 18(5) 929-941.

[10] Gao W., Yang L., Zhang X. and Liu H. 2010. An Improved Sobel Edge Detection. IEEE international conference on computer science and information technology (ICCSIT), vol. 5, 67-71.

[11] Ziou D. and Tabbone S. 1998. Edge Detection Techniques - An Overview. International Journal of Pattern Recognition and Image Analysis, vol. 8,537-559.

\section{AUTHOR PROFILE}

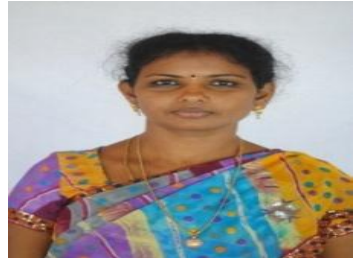

Dr.V.S. Manjula has Completed MCA, M.Phil., B.Ed., Ph.D and She received Ph.D degree in Computer Science from Bharathiar University in 2013. She worked in Head in MCA Department, Gurushree Shantivijai Jain College, Chennai. At present she is working in Associate Professor in Computer Science and Engineering Department in St. Joseph University College of Engineering \& Technology, Dar-Es-Salaam in Tanzania, East Africa. She is a Research Supervisor in Information \& Communication Technology (ICT) in St. Eugene University in Zambia and she is a member of Research Journal of International Association of Computer Science \& Information Technology (IACSIT) \&International Association of Engineers (IAENG) Member Number: 143718 - USA. She has published more than 13 International Journals and National \& International Conferences

Dr. V.S. Manjula. "Image Edge Detection and Segmentation by using Histogram Thresholding method ." International Journal of Engineering Research and Applications (IJERA) 7.8 (2017): $10-16$. 